\title{
Some Classes of $k$-Uniformly Functions with Bounded Radius Rotation
}

\author{
Khalida Inayat Noor*, Rabia Fayyaz and Muhammad Aslam Noor \\ Mathematics Department, COMSATS Institute of Information Technology, Park Road, Islamabad, Pakistan
}

Received: 14 Mar. 2013, Revised: 14 Jul. 2013, Accepted: 16 Jul. 2013

Published online: 1 Mar. 2014

\begin{abstract}
We use Ruscheweyh derivative to define certain new classes of analytic functions with bounded radius rotation and related to conic domains. Some interesting and significant results such as inclusion results, growth rate of coefficients and radius problems for these new classes of $k$-uniformly functions. Several special cases are discussed. Results obtained in this paper may stimulate further research activities in this field.
\end{abstract}

Keywords: Convex, starlike, conic domains, subordinate, Bernardi operator, bounded radius rotation, convolution, radius of convexity. 2010 AMS Subject Classification: 30C45, 30C50, 30C55

\section{Introduction}

Let $A$ be the class of functions of the form

$f(z)=z+\sum_{j=2}^{\infty} a_{j} z^{j}$

which are analytic in the open unit disc $E=\{z:|z|<1\}$.

Let $S^{*}(\delta)$ and $C(\delta)$ denote the subclasses of $A$ consisting of starlike and convex functions of order $\delta(0 \leq \delta<1)$ respectively, where $S^{*}(0)=S^{*}$ and $C(0)=C$ are the well known classes of starlike and convex functions. Let $S \subset A$ be the class of univalent functions. Then the inclusion relation $C \subset S^{*} \subset S$ holds, e.g. see [3].

Kanas and Wisniowska [5] studied the classes of $k$-uniformly convex functions, denoted by $k-U C V$, and the corresponding class $k-S T$ related by the Alxander type relation. See also [10].

Let $f, g \in A, g(z)=z+\sum_{j=2}^{\infty} b_{j} z^{j}$ and $f(z)$ given by (1.1). Then the convolution (Hadamard product) of $f$ and $g$ is defined by

$(f * g)(z)=z+\sum_{j=2}^{\infty} a_{j} b_{j} z^{j}=(g * f)(z), z \in E$.

Also, if $f$ and $g$ are analytic in $E$, we say that $f$ is subordinate to $g$ in $E$, written as $f \prec g$ or $f(z) \prec g(z)$, if there exists a Schwarz function $w(z)$ such that $f(z)=g(w(z))$ for $z \in E$.

For $k \in[0, \infty)$, the conic domain $\Omega_{k}$ are defined as follows, see [4].

$\Omega_{k}=\left\{u+i v: u>k \sqrt{(u-1)^{2}+v^{2}}\right\}$.

For fixed $k, \Omega_{k}$ represents the conic regions bounded successively, by the imaginary axis $(\mathrm{k}=0)$, the right branch of a hyperbola $(0<k<1)$ and a parabola $k=1$.

When $k>1$, the domain becomes a bounded domain being interior of the ellipse. We shall consider here $k \in$ $[0,1]$.

Related to the domains $\Omega_{k}$, the following functions $p_{k}(z)$ play the role of extermal functions mapping $E$ onto $\Omega_{k}$. These functions are univalent in $E$ and belong to the class $P$ of functions with positive real part and are given as:

$p_{k}(z)=\left\{\begin{array}{l}\frac{1+z}{1-z},(k=0), \\ 1+\frac{2}{\pi^{2}}\left(\log \frac{1+\sqrt{z}}{1-\sqrt{z}}\right)^{2}, \quad(k=1), \\ 1+\frac{2}{1-k^{2}} \sinh ^{2}\left[\left(\frac{2}{\pi} \arccos k\right) \arctan \sqrt{(z)}\right], \quad(0<k<1) .\end{array}\right.$

Using subordination concept, we define the class $P\left(p_{k}\right)$ as follows.

Let $p(z)$ be analytic in $E$ with $p(0)=1$. Then $p \in P\left(p_{k}\right)$ if $p(z) \prec p_{k}(z), k \in[0,1]$ and $p_{k}(z)$ are given by (1.3).

\footnotetext{
*Corresponding author e-mail: khalidanoor@ hotmail.com
} 
It is known [5] that $p \in P\left(p_{k}\right)$ is in the class $P(\delta)$ of functions with positive real part greater than $\delta=\frac{k}{k+1}$. That is $P\left(p_{k}\right) \subset P\left(\frac{k}{k+1}\right) \subset P$.

We generalize the class $P\left(p_{k}\right)$ as follows.

Definition 1. Let $p$ be analytic in $E$ with $p(0)=1$. Then $p \in P_{m}\left(p_{k, \alpha}\right), m \geq 2,0 \leq \alpha<1, k \in[0,1]$, if and only if we can write

$$
\begin{aligned}
p(z) & =\left(\frac{m}{4}+\frac{1}{2}\right)\left\{(1-\alpha) p_{1}(z)+\alpha\right\} \\
& -\left(\frac{m}{4}-\frac{1}{2}\right)\left\{(1-\alpha) p_{2}(z)+\alpha\right\},
\end{aligned}
$$

where $p_{i} \in P\left(p_{k}\right), i=1,2$.

Also, it is obvious that $p \in P_{m}\left(p_{k, \alpha}\right)$ can be written as $p(z)=(1-\alpha) h(z)+\alpha, h \in P_{m}\left(p_{k, 0}\right)=P_{m}\left(p_{k}\right)$ in $E$.

For $m=2, \alpha=0$, we have the class $P\left(p_{k}\right)$. When $k=0$, the class $P_{m}\left(p_{0, \alpha}\right)$ reduces to $P_{m}(\alpha)$, see [11], and $P_{m}(0)=P_{m}$ was introduced in [12].

The relation (4) can be expressed as:

$$
\begin{aligned}
p(z) & =\left(\frac{m}{4}+\frac{1}{2}\right)\left(p_{1}(z) * \phi_{\alpha}(z)\right) \\
& -\left(\frac{m}{4}-\frac{1}{2}\right)\left(p_{2}(z) * \phi_{\alpha}(z)\right),
\end{aligned}
$$

where

$\phi_{\alpha}(z)=\frac{1-(1-2 \alpha) z}{1-z}, \Re \phi_{\alpha}(z) \geq \alpha, z \in E$,

and $p_{i} \prec p_{k}(z), i=1,2$.

For $n \in N_{\circ}=\{0,1,2,3 \ldots\}$, let $D^{n}: A \rightarrow A$ be the operator defined by

$D^{n} f(z)=\frac{z}{(1-z)^{n+1}} * f(z)$,

so that

$$
\begin{aligned}
D^{n} f(z) & =\frac{z\left(z^{n-1} f(z)\right)^{n}}{n !} \\
& =z+\sum_{j=2}^{\infty} \frac{(j+n-1) !}{n !(j-1) !} a_{j} z^{j} .
\end{aligned}
$$

The following identity holds and can easily be verified.

$z\left(D^{n} f(z)\right)^{\prime}=(n+1) D^{n+1} f(z)-n D^{n} f(z)$.

The operator $D^{n}$ is called Ruscheweyh derivative of order $n$, see [16].

We shall assume, unless otherwise stated, that $n \in N_{\circ}, m \geq 2, k \in[0,1], 0 \leq \alpha<1$ and $z \in E$.

A function $f \in A$ is said to belong to the class $R_{m}(n, \alpha)$ if and only if

$$
\frac{z\left(D^{n} f(z)\right)^{\prime}}{D^{n} f(z)} \in P_{m}(\alpha), \quad z \in E .
$$

When $n=0, \alpha=0$, we get the class $R_{m}$ of functions with bounded radius rotation, see [3].

We now define the following.

Definition 2. For $n \in N_{\circ}, m \geq 2,0 \leq \alpha<1, k \in[0,1], a$ function $f \in A$ is said to belong to the class $k-U R_{m}(n, \alpha)$ if and only if

$$
\frac{z\left(D^{n} f(z)\right)^{\prime}}{D^{n} f(z)} \in P_{m}\left(p_{k}, \alpha\right)
$$

for $z \in E$.

As special cases, we have the following:

$$
\begin{aligned}
& \text { i. } 0-U R_{m}(k, \alpha)=R_{m}(k, \alpha) \text {. } \\
& \text { ii. } 0-U R_{m}(0,0)=R_{m} . \\
& \text { iii. } k-U R_{2}(0,0)=k-S T .
\end{aligned}
$$

By using Alexander type relation, the class $k-U V_{m}(n, \alpha)$ is defined as follows.

Let $f \in A$. Then

$$
f \in k-U V_{m}(n, \alpha)
$$

if and only if

$$
z f^{\prime} \in k-U R_{m}(n, \alpha) \in E
$$

We note that

i. $0-U V_{m}(n, \alpha)=V_{m}(n, \alpha)$.

ii. For $k=0, n=\alpha=0$, we have the class $k-U V_{2}(0,0)=k-U C V$.

$V_{m}$ of the functions with bounded boundary rotation, see [3].

For a function $f \in A$, we define the integral operator $I_{c}$ : $A \rightarrow A$, by

$I_{c}(f)=\frac{c+1}{z^{c}} \int_{0}^{z} t^{c-1} f(t) d t, \quad(\Re c>-1)$.

When $c \in N=\{1,2,3, \ldots\}$, the operator $I_{c}$ was introduced by Bernadi [2]. In particular $I_{1}$, was studied earlier by Libera [6] and Livingston [7].

\section{Preliminaries}

In order to derive our main results, we need the following lemmas.

Lemma 1([14], p 217). Let $\beta>0, \gamma \geq 0$ and let $h(z)$ be analytic in $E$ with $h(0)=1$. Then

$$
\left(h(z) * \frac{\phi_{\beta, \gamma}(z)}{z}\right)=h(z)+\frac{z h^{\prime}(z)}{\beta h(z)+\gamma},
$$

where

$\phi_{\beta, \gamma}(z)=\sum_{j=1}^{\infty}\left(\frac{\beta+\gamma}{j \beta+\gamma}\right) z^{j}$. 
Lemma 2. Let $k \geq 0$ and let $\sigma, \delta$ be any complex numbers with $\sigma \neq 0$ and $0 \leq \alpha<\Re\left(\frac{\sigma k}{k+1}+\delta\right)$. If $h(z)$ is analytic in $E, h(0)=1$ and satisfies

$\left(h(z)+\frac{z h^{\prime}(z)}{\sigma h(z)+\delta}\right) \prec p_{k, \alpha}(z)$,

and $q_{k, \alpha}(z)$ is an analytic solution of

$q_{k, \alpha}(z)+\frac{z q_{k, \alpha}^{\prime}(z)}{\sigma q_{k, \alpha}(z)+\delta}=P_{k, \alpha}(z)$,

then $q_{k, \alpha}(z)$ is univalent, and

$h(z) \prec q_{k, \alpha}(z) \prec p_{k, \alpha}(z)$.

The function $q_{k, \alpha}(z)$ is the best dominant of (10) and is given as

$q_{k, \alpha}(z)=\left[\int_{0}^{1}\left(\exp \int_{t}^{t z} \frac{p_{k, \alpha}(u)-1}{u} d u\right) d t\right]^{-1}-\frac{\delta}{\sigma}$.

This result can be found in [10] and is an easy generalization of one due to Kanas [4].

Lemma 3([13]). Let $f \in C$ and $g \in S^{*}$. Then, for every function $F$ analytic in $E$ with $F(0)=1$, we have

$$
\left(\frac{f * F g}{f * g}\right)(E) \subset \bar{C} o(F(E)),
$$

where $\bar{C} o(F(E))$ denotes the closed convex hull of $F(E)$.

Lemma 4([15]). Let $p$ be an analytic function in $E$ with $p(0)=1$ and $\Re p(z>0), z \in E$. Then, for $s>0$ and $v \neq-1$ (Complex),

$$
\mathfrak{R}\left\{p(z)+\frac{s z p^{\prime}(z)}{p(z)+v}\right\}>0
$$

for $|z|<r_{0}$, where $r_{0}$ is given by

$$
\begin{gathered}
r_{0}=\frac{|v+1|}{\sqrt{A+\left(A^{2}-\left|v^{2}-1\right|^{2}\right)^{\frac{1}{2}}}}, \\
A=2(s+1)^{2}+\left|v^{2}\right|-1
\end{gathered}
$$

and this radius is best possible.

\section{Main Results}

Theorem 1. $k-U R_{m}(n+1, \alpha) \subset k-U R_{m}(n, \alpha)$ for each $n \in N_{0}$.

Proof. Let $f \in k-U R_{m}(n+1, \alpha)$. Then, for $z \in E$,

$$
\frac{z\left(D^{n+1} f(z)\right)^{\prime}}{D^{n+1} f(z)} \in P_{m}\left(p_{k, \alpha}\right) \text {. }
$$

Set

$$
\frac{z\left(D^{n} f(z)\right)^{\prime}}{D^{n} f(z)}=H(z) .
$$

$H(z)$ is analytic in $E$ and $H(0)=1$.

From (6) and (11), we obtain

$\frac{z\left(D^{n+1} f(z)\right)^{\prime}}{D^{n+1} f(z)}=\left\{H(z)+\frac{z H^{\prime}(z)}{H(z)+n}\right\} \in P_{m}\left(p_{k, \alpha}\right)$ in $E$

Let

$H(z)=\left(\frac{m}{4}+\frac{1}{2}\right) h_{1}(z)-\left(\frac{m}{4}-\frac{1}{2}\right) h_{2}(z)$.

Using (12), (13) and Lemma 1 with $\alpha=1, c=n$, it follows that

$\left(h_{i}(z)+\frac{z h_{i}^{\prime}(z)}{h_{i}(z)+n}\right) \in P\left(p_{k, n}\right), \quad i=1,2, z \in E$.

Applying Lemma 2, we obtain $h_{i} \in P\left(p_{k, \alpha}\right)$ in $E$, for $i=$ 1,2 and consequently $H \in P_{m}\left(p_{k, \alpha}\right)$ in $E$. This proves that $f \in k-U R_{m}(n, \alpha)$ in $E$.

Corollary 1. Let $k=0$. Then $f \in R_{m}(n+1, \alpha)$ implies that $f \in R_{m}(n, \sigma)$, where $\sigma$ is given by

$\sigma=\left[\frac{n+1}{{ }_{2} F_{1}\left(2(1-\alpha), 1, n+2 ; \frac{1}{2}\right)}-n\right]$,

and ${ }_{2} F_{1}$ represents Gauss hypergeometric function. This result is sharp.

Proof. In fact, from (14), it follows that

$$
\left(h_{i}(z)+\frac{z h_{i}^{\prime}(z)}{h_{i}(z)+n}\right) \in P(\alpha), \quad i=1,2 .
$$

This implies, by using a result due to Miller-Mocann [8, p 113 , Theorem 3.3 e] that $h_{i} \in P(\sigma)$, where $\sigma$ is given by (15).

For sharpness, the extremal function is given as

$$
p_{0}(z)=\left(\frac{1}{g(z)}-n\right)
$$

with

$$
\begin{aligned}
g(z) & =\int_{0}^{1}\left(\frac{1-z}{1-t z}\right)^{2(1-\alpha)} t^{n} d t \\
& =\frac{{ }_{2} F_{1}\left(2(1-\alpha), 1, n+2, \frac{z}{z-1}\right)}{n+1} .
\end{aligned}
$$

Consequently $H \in P_{m}(\sigma), \sigma$ is given by (15). This completes the proof that $f \in R_{m}(n, \sigma)$ in $E$. 
As a special case, when $n=0$, we note that $R_{m}(1, \alpha)=$ $V_{m}(\alpha)$, where $V_{m}(\alpha)$ is the class of functions of bounded boundary rotation with order $\alpha$, see [3], and $f \in V_{m}(\alpha)$ implies that $f \in R_{m}(0, \sigma)=R_{m}\left(\sigma_{0}\right)$, where $R_{m}\left(\sigma_{0}\right)$ is the corresponding class of bounded radius rotation with order $\sigma_{0}$, order $\sigma_{0}$ is given by (15) with $n=0$, as follows.

$\sigma_{0}=\sigma_{0}=\left[{ }_{2} F_{1}\left(2(1-\alpha), 1 ; 2, \frac{1}{2}\right)\right]^{-1}$

$$
=\left\{\begin{array}{l}
\frac{1-2 \alpha}{2^{2(1-\alpha)\left(1-2^{2 \alpha-1}\right)}}, \quad\left(\alpha=\frac{1}{2}\right) . \\
\frac{1}{2 \ln 2}, \quad\left(\alpha=\frac{1}{2}\right)
\end{array} .\right.
$$

When $\alpha=0$, we have

$$
\sigma_{0}=\frac{1}{2^{2}\left(1-\frac{1}{2}\right)}=\frac{1}{2} .
$$

This shows, if $f \in V_{m}$, then $f \in R_{m}\left(\frac{1}{2}\right)$. When $m=2$, this leads to a well known result that a convex univalent function is a starlike function of order $\frac{1}{2}$.

Since $P\left(p_{k, \alpha}\right) \subset P\left(\frac{k+\alpha}{k+1}\right)$, we have the following result on the rate of growth of coefficients for $f \in R_{m}(n, \gamma), \gamma=$ $\frac{k+\alpha}{k+1}$.

Theorem 2. Let $f \in R_{m}(n, \gamma), \gamma=\frac{k+\alpha}{k+1}$ and be given by (1). Then, for $j>3, m \geq 2$.

$$
a_{j}=O(1) \cdot j^{\left\{(1-\gamma)\left(\frac{m}{2}+1\right)-(n+1)\right\}},
$$

where $O(1)$ is a constant depending on $m, k, \alpha$ and $n$. The exponent $\left\{(1-\gamma)\left(\frac{m}{2}+1\right)-(n+1)\right\}$ is best possible.

Proof.

$$
\begin{aligned}
D^{n} f(z) & =\frac{z}{(1-z)^{n+1} * f(z)} \\
& =\left[z+\sum_{j=2}^{\infty} \frac{(j+n-1) !}{n !(j-1) !} z^{j}\right] *\left[z+\sum_{j=2}^{\infty} a_{j} z^{j}\right] \\
& =z+\sum_{j=2}^{\infty} \frac{(j+n-1) !}{n !(j-1) !} a_{j} z^{j} .
\end{aligned}
$$

Now, since $D^{n} f \in V_{m}(\gamma)$ if and only if $\left(D^{n} f\right)^{\prime} \in R_{m}(\gamma)$, we use a coefficient result for the class $V_{m}(\gamma)$ proved in [9] to have, for $j>3, m \geq 2$.

$$
\begin{aligned}
& \frac{(j+n-1) !}{n !(j-1) !}\left|a_{j}\right| \\
& <\left\{m^{2}(1-\gamma)^{2}+m(1-\gamma)\right\} 2^{1-2 \gamma}\left(\frac{2}{3} j\right)^{(1-\gamma)\left(\frac{m}{2}+1\right)-1},
\end{aligned}
$$

and this gives us

$$
a_{n}=O(1) \cdot j^{\left\{(1-\gamma)\left(\frac{m}{2}+1\right)-(n+1)\right\}}, \quad j>3, m \geq 2 .
$$

The function $F_{0} \in R_{m}(n, \gamma)$ defined by

$$
D^{n} F_{0}(z)=\frac{z\left(1+\delta_{1} z\right)^{\left(\frac{m}{2}-1\right)(1-\gamma)}}{\left(1-\delta_{2} z\right)^{\left(\frac{m}{2}+1\right)(1-\gamma)}}, \quad\left|\delta_{1}\right|=\left|\delta_{2}\right|=1,
$$

shows that the exponent $\left\{(1-\gamma)\left(\frac{m}{2}+1\right)-(n+1)\right\}$ is the best possible.

Theorem 3. $\bigcap_{n=0}^{\infty} R_{m}(n, \gamma)=\{i d\}, \quad \gamma=\frac{k+\alpha}{k+1}$, where id is the identity function.

Proof. Let $f(z)=z$. Then it follows trivially that $z \in R_{m}(n, \gamma)$ for $n \in N_{0}$. On the contrary, assume that $f \in \bigcap_{n=0}^{\infty} R_{m}(n, \gamma)$ with $f(z)$ given by (1). Then, from Theorem 2, we deduce that $f(z)=z$.

We now show that the class $k-U R_{m}(n, \alpha)$ is preserved under generalized Bernardi operator given by (7).

Theorem 4. Let $f \in k-U R_{m}(n, \alpha)$ and let $I_{c}(f), \Re_{c}>-1$, be defined by (7). Then, for $z \in E, I_{c}(f) \in k-U R_{m}(n, \alpha)$.

Proof. Let

$$
\begin{aligned}
& \frac{z\left(D^{n} I_{c}(f(z))\right)^{\prime}}{D^{n} I_{c}(f(z))} \\
& =h(z)=\left(\frac{m}{4}+\frac{1}{2}\right) p_{1}(z)-\left(\frac{m}{4}-\frac{1}{2}\right) p_{2}(z),
\end{aligned}
$$

where $h(z)$ is analytic in $E, h(0)=1$.

Simple computations and use of (7), (16), lead to the following.

$\frac{z\left(D^{n} f(z)\right)^{\prime}}{D^{n} f(z)}=\left(h(z)+\frac{z h^{\prime}(z)}{h(z)+c}\right) \in P_{m}\left(p_{k, \alpha}\right)$.

With similar technique used in Theorem 1, and from (16), (17), it follows that

$$
\left(p_{i}(z)+\frac{z p_{i}^{\prime}(z)}{p_{i}(z)+c}\right) \in P\left(p_{k, \alpha}\right), z \in E, i=1,2 .
$$

We now apply Lemma 2 to have

$$
p_{i} \in P\left(p_{k, \alpha}\right), \quad i=1,2,
$$

and consequently $h \in P_{m}\left(p_{k, \alpha}\right), z \in E$. This proves $I_{c}(f(z)) \in k-U R_{m}(n, \alpha)$.

As special cases, we note that:

i. For $m=2 n=0=\alpha$, the class $k-S T$ is preserved under generalized Bernardi operator.

ii. With $m=2, n=1, \alpha=0$, it follows from Theorem 4, that the class $k-U C V$ of uniformly convex functions is invariant under the operator given by (17).

Corollary 2. Let $f \in k-U R_{m}(n, \alpha) \subset R_{m}\left(n, \alpha_{1}\right)$, $\alpha_{1}=\frac{k+\alpha}{k+1}$. Then $I_{c}(f) \in R_{m}(n, \beta)$, where $c>-1$, and

$\beta=\left\{\frac{c+1}{{ }_{2} F_{1}\left(2\left(1-\alpha_{1}\right), 1 ; c+2 ; \frac{1}{2}\right)-c}\right\}$. 
Proof. Proceeding as in Theorem 4, it follows from (17) that

$$
\left(p_{i}(z)+\frac{z p_{i}^{\prime}(z)}{p_{i}(z)+c} \in P\left(\alpha_{1}\right)\right), \alpha=\frac{k+\alpha}{k+1} .
$$

Then, as in corollary $1, p_{i} \in P(\beta), i=1, z \in E$, where $\beta$ is given by (18). Consequently, from (18), $h \in P_{m}(\beta)$ and hence $I_{c}(f) \in R_{m}(n, \beta), z \in E$.

\section{Corollary 3.}

i. Let $\alpha=0, k=1$ and $c=0$. Then $f \in 1-U R_{m}(n, 0) \subset$ $R_{m}\left(n, \frac{1}{2}\right)$. Then, from Theorem $4, I_{0}(f) \in R_{m}\left(n, \beta_{0}\right)$, where $\beta_{0}=\frac{1}{{ }_{2} F_{1}\left(1,1,2, \frac{1}{2}\right)}=\frac{1}{2 \ln 2}$.

ii. For $c=1, k=0=\alpha, I_{1}(f) \in R_{m}\left(n, \beta_{1}\right)$, where

$$
\beta_{1}=\left\{\frac{2}{{ }_{2} F_{1}\left(2,1 ; 3, \frac{1}{2}\right)}-1\right\}=\frac{1}{2(2 \ln 2-1)} .
$$

iii. For $c=1, \quad k=1, \quad \alpha=0$, $f \in 1-U R_{m}(n, 0) \subset R_{m}\left(n, \frac{1}{2}\right)$. This implies, $I_{1}(f) \in R_{m}\left(n, \beta_{2}\right)$, where

$$
\begin{aligned}
\beta_{2} & =\left\{\frac{2}{{ }_{2} F_{1}\left(1,1 ; 3, \frac{1}{2}\right)-1}\right\} \\
& =\left(\frac{1}{2(1-\ln 2)}-1\right) \approx 0.629 .
\end{aligned}
$$

Theorem 5. Let $f \in k-U R_{m}(n, \alpha)$. Then $I_{n}(f) \in k-U R_{m}(n+1, \alpha)$, where $I_{n}(f)$ is defined by (7) with $c=n \in N_{0}$. The proof is straightforward when we note, from (6) and (7), that

$$
D^{n} f(z)=D^{n+1} I_{n} f(z) .
$$

Next we consider the converse of the problem involving the operator (7) for the case when $m=2$.

Theorem 6. Let $I_{c}(f)$, defined by (7), belong to $k-U R_{2}(n, \alpha)$. Then $f \in k-U R_{2}(n, \alpha)$ for $|z|<r_{c}$, where

$$
r_{c}=\left\{\begin{array}{l}
\frac{2-\sqrt{3+c^{2}}}{1-c}, \quad(c \neq 1), \\
\frac{1}{2}, \quad(c=1),
\end{array}\right.
$$

and this radius is best possible.

Proof. We first prove the following.

Let $f_{1} \in k-U R_{2}(n, \alpha)$ and let $\phi(z)$ be convex univalent in $E$. Then we show that $\left(\phi(z) * f_{1}(z)\right) \in k-U R_{2}(n, \alpha)$ in $E$.

Now

$$
\begin{aligned}
\frac{z\left(D^{n}\left(\phi * f_{1}\right)\right)^{\prime}}{D^{n}\left(\phi * f_{1}\right)}=\frac{z\left(\left(\phi * D^{n} f_{1}\right)\right)^{\prime}}{\left(\phi * D^{n} f_{1}\right)} & =\frac{\phi * \frac{z\left(\left(D^{n} f_{1}\right)\right)^{\prime}}{\left(D^{n} f_{1}\right)} \cdot D^{n} f_{1}}{\phi * D^{n} f_{1}} \\
& =\frac{\phi * F\left(D^{n} f_{1}\right)}{\phi * D^{n} f_{1}}
\end{aligned}
$$

Since $f_{1} \in k-U R_{2}(n, \alpha), \quad D^{n} f_{1} \in S^{*}\left(\frac{k+\alpha}{k+1}\right) \subset S^{*}$, $F \in P\left(p_{k, \alpha}\right)$ and $\phi \in C$ in $E$, we use Lemma 3 to conclude that $\phi * f_{1} \in k-U R_{2}(n, \alpha)$.

We define $h_{c}(z)$ by

$h_{c}(z)=\sum_{j=1}^{\infty} \frac{j+c}{1+c} z^{j}=\frac{z-\left[\frac{c}{1+c}\right] z^{2}}{(1-z)^{2}}$.

Now, from (17), we can write

$$
f_{1}(z)=I_{c}(f)=\frac{c+1}{z^{c}} \int_{0}^{z} t^{c-1} f(t) d t \quad(c>-1) .
$$

Then

$$
f(z)=\frac{c I_{c}(f)+z\left(I_{c}(f)\right)^{\prime}}{c+1}=I_{c}(f) * h_{c}(z),
$$

where $h_{c}(z)$ is given by (20).

It is known [1] that $h_{c}(z)$ is convex for $|z|<r_{c}$ where $r_{c}$ is given by (19). Since $I_{c}(f) \in k-U R_{2}(n, \alpha)$ in $E$ and $h_{c}$ is convex for $|z|<r_{c}$, it follows that for $f \in k-U R_{2}(n, \alpha)$ for $|z|<r_{c}$.

As special cases, we note that:

i. For $c=1, I_{1}(f)$ is Libere-Livingston operator and $I_{1}(f) \in k-U R_{2}(n, \alpha)$ in $E$ implies that $f \in k-U R_{2}(n, \alpha)$ for $|z|<\frac{1}{2}$.

ii. Alexander operator $I_{0}(f) \in k-U R_{2}(n, \alpha)$ in $E$ implies that $f \in k-U R_{2}(n, \alpha)$ for $|z|<2-\sqrt{3}$.

Remark 1. Let $f_{n}(z)=\frac{z}{(1-z)^{n+1}}, n \in N_{0}$. Then it can easily be verified that $f_{n} \in C$ for $|z|<r_{n}$, where

$$
r_{n}=\frac{2}{(3 n+1)+\sqrt{(3 n+1)^{2}-4 n^{2}}} .
$$

We have:

Theorem 7. Let $D^{n}(f) \in k-U R_{2}(\alpha)=k-S T(\alpha)$ in $E$. Then $f \in k-S T(\alpha)$ for $|z|<r_{n}$, where $r_{n}$ is given by (21).

Proof. Since $D^{n}(f) \in k-U R_{2}(\alpha)=k-S T(\alpha)$, it follows that $\left(\frac{z}{(1-z)^{n+1}} * f(z)\right) \in k-S T(\alpha)$.

Now $\frac{z}{(1-z)^{n+1}}$ is convex for $|z|<r_{n}$ and $k-S T(\alpha)$ is closed under convex convolution, we obtain the required result.

Theorem 8. Let $I_{n}(f)$ be defined by (7) with $c=n \in N_{0}$ and let $I_{n} f \in k-U R_{2}(n, \alpha)$. Then, for $\gamma=\frac{k+\alpha}{k+1}, D^{n} f \in S^{*}(\gamma)$ for $|z|<r_{\gamma}$ where the exact value of $r_{\gamma}$ will be given in the proof.

Proof. Proceeding as in Theorem 4, with $n=c$, we have

$\frac{z\left(D^{n} f(z)\right)^{\prime}}{D^{n} f(z)}=h(z)+\frac{z h^{\prime}(z)}{h(z)+n}$, 
where $h(z)=\frac{z\left(D^{n} I_{n} f(z)\right)^{\prime}}{D^{n} I_{n}(f(z))} \in P\left(p_{k, \alpha}\right) \subset P(\gamma)$ in E. From (22), we have with $h(z)=(1-\gamma) h_{0}(z)+\gamma$.

$$
\frac{1}{1-\gamma}\left\{\frac{z\left(D^{n} f(z)\right)^{\prime}}{D^{n} f(z)}-\gamma\right\}=h_{0}(z)+\frac{\frac{1}{1-\gamma} \cdot z h_{0}^{\prime}(z)}{h_{0}(z)+\frac{n+\gamma}{1-\gamma}}, h_{0} \in P
$$

We use Lemma 4 with $s=\frac{1}{1-\gamma}, v=\frac{n+\gamma}{1-\gamma} \neq-1$, to have

$\Re\left[\frac{1}{1-\gamma}\left\{\frac{z\left(D^{n} f(z)\right)^{\prime}}{D^{n} f(z)}-\gamma\right\}\right] \geq 0, \quad$ for $|z|<r_{\gamma}$,

where

$$
\begin{gathered}
r_{\gamma}=\frac{|v+1|}{\sqrt{A+\left(A^{2}-\left|v^{2}-1\right|^{2}\right)^{\frac{1}{2}}}}, \\
A=2(s+1)^{2}+|v|^{2}-1, \\
s=\frac{1}{1-\gamma}, \\
v=\frac{n+\gamma}{1-\gamma} .
\end{gathered}
$$

This completes the proof.

Remark 2. We can use well known distortion results for the class $P$, see[3], to have

$$
\begin{aligned}
& \Re\left[\frac{1}{1-\gamma}\left\{\frac{z\left(D^{n} f(z)\right)^{\prime}}{D^{n} f(z)}-\gamma\right\}\right] \\
& \geq \Re h_{0}(z)\left\{1-\frac{2 \gamma}{1-r^{2}} \frac{1}{(1-\gamma) \frac{1-r}{1+r}+(n+r)}\right\}
\end{aligned}
$$

That is,

$$
\begin{aligned}
& \Re\left[\frac{1}{1-\gamma}\left\{\frac{z\left(D^{n} f(z)\right)^{\prime}}{D^{n} f(z)}-\gamma\right\}\right] \\
& \geq \Re h_{0}(z)\left\{\frac{(1-2 \gamma-n) r^{2}-2(2-\gamma) r+(n+1)}{(1-\gamma)(1-r)^{2}+(n+\gamma)\left(1-r^{2}\right)}\right\},
\end{aligned}
$$

and right hand side is positive for $r<r_{*}$, where

$$
r_{*}=\frac{n+1}{(2-\gamma)+\sqrt{(2-\gamma)^{2}-(n+1)(1-2 \gamma)-n}} .
$$

This shows that for $D^{n} f \in S^{*}(\gamma)$ for $|z|<r_{*}$, and $r_{*}$ is given by (24).

\section{Acknowledgement}

The authors are grateful to Dr. S. M. Junaid Zaidi, Rector, COMSATS Institute of Information Technology, Pakistan for providing excellent research and academic environment.

\section{References}

[1] R. W. Barnard, C. Kellogg, Applications of Convolution operators to problems in Univalent Function Theory, Michigan Math. J., 27, 81-94 (1980).

[2] S. Bernardi, Convex and starlike univalent functions, Trans. Amer. Math. Soc., 135, 429-446 (1969).

[3] A. W. Goodman, Univalent Functions, Polygonal Publishing House, Washington, New Jersey, I,II, (1983).

[4] S. Kanas, Techniques of differential sunordination for domains bounded by conic sections, Internat. J. Math. Math. Sci., 38, 2389-2400 (2003).

[5] S. Kanas, A. Wisniowska, Conic regions and k-uniform convexity, J. Comput. Appl. Math., 105, 327-336 (1999).

[6] R. J. Libera, Some classes of regular univalent functions, Proc. Amer. Math. Soc., 16, 735-758 (1965).

[7] A. W. Livingston, On the radius of univalence of certain analytic functions, proc. Amer. Math. Soc., 17, 352-357 (1966).

[8] S. S. Miller, P.T. Mocanu, Differential Subordinations: Theory and Applications, series on Monographs and Textbooks in Pure and Applied Mathematics, Marcel Dekker Inc., New York, Basel, 225, (2000).

[9] K. I. Noor, On subclasses of close-to-convex functions of higher order, Internat. J. Math. Math. Sci. , 15, 279-290 (1992).

[10] K. I. Noor, M. Arif, Wasim-ul-Haq, On k-uniformly close-to-concex functions of complex order, Appl. Math. Comput., 215, 629-635 (2009).

[11] K. S. Padmanabhan, R. Parvatham, Properties of a class of functions with bounded boundary rotation, Ann. Polon. Math., 31, 311-323 (1975).

[12] B. Pinchuk, Functions of bounded boundary rotation, Israel J. Math., 10, 7-16 (1971).

[13] S. Ruscheweyh, Convolution in Geometric Function Theory, Les Presse. De universite de Montreal, Montreal, (1982).

[14] S. Ruscheweyh, Ein Invarianzeign- shaft der BazilevicFunkionen, Math. Z., 134, 215-219 (1973).

[15] S. Ruscheweyh, V. Singh, On certain extremal problems for functions with positive real parts, Amer. Math. Soc., 61, 329-334 (1976).

[16] S. Ruscheweyh, A new criteria for univalent functions, Proc. Amer. Math. Soc., 49, 109-115 (1975). 


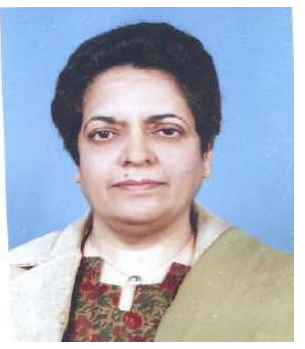

Khalida Inayat Noor is a leading world-known figure in mathematics and is presently employed as HEC Foreign Professor at CIIT, Islamabad. She obtained her $\mathrm{PhD}$ from Wales University (UK). She has a vast experience of teaching and research at university levels in various countries including Iran, Pakistan, Saudi Arabia, Canada and United Arab Emirates. She was awarded HEC best research paper award in 2009 and CIIT Medal for innovation in 2009. She has been awarded by the President of Pakistan: Presidents Award for pride of performance on August 14, 2010 for her outstanding contributions in mathematical sciences and other fields. Her field of interest and specialization is Complex analysis, Geometric function theory, Functional and Convex analysis. She introduced a new technique, now called as Noor Integral Operator which proved to be an innovation in the field of geometric function theory and has brought new dimensions in the realm of research in this area. She has been personally instrumental in establishing $\mathrm{PhD} / \mathrm{MS}$ programs at CIIT. Dr. Khalida Inayat Noor has supervised successfully several Ph.D and MS/M.Phil students. She has been an invited speaker of number of conferences and has published more than 400 (Four hundred ) research articles in reputed international journals of mathematical and engineering sciences. She is member of editorial boards of several international journals of mathematical and engineering sciences.

Rabia Fayyaz is lecturer in Department of Mathematics, COMSATS Institute of Information and Technology, Islamabad, Pakistan. Her field of interests are Complex Analysis, Geometric Function Theory and related areas.

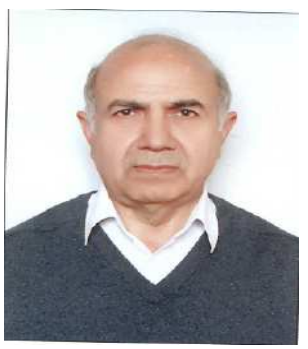

Muhammad
Aslam Noor earned his $\mathrm{PhD}$ degree from Brunel University, London, UK (1975) in the field of Applied Mathematics(Numerical Analysis and Optimization). $\mathrm{He}$ has vast experience of teaching and research at university levels in various countries including Pakistan, Iran, Canada, Saudi Arabia and United Arab Emirates. His field of interest and specialization is versatile in nature. It covers many areas of Mathematical and Engineering sciences such as Variational Inequalities, Operations Research and Numerical Analysis. He has been awarded by the President of Pakistan: President's Award for pride of performance on August 14, 2008, in recognition of his contributions in the field of Mathematical Sciences. He was awarded HEC Best Research paper award in 2009. He has supervised successfully several Ph.D and MS/M.Phil students. He is currently member of the Editorial Board of several reputed international journals of Mathematics and Engineering sciences. He has more than 750 research papers to his credit which were published in leading world class journals. 